\title{
Buenas prácticas en la educación superior virtual a partir de especificaciones de estándares e-Learning*
}

\section{Good practices in virtual higher education based on specifications for e-Learning standards}

\section{Boas práticas no ensino superior virtual a partir de especificações de padrões e-Learning}

Edgar Javier Carmona Suárez** Elizabeth Rodríguez Salinas***

\begin{abstract}
**Doctor en TIC. Profesor titular en la Universidad del Quindío, Grupo de investigación GEDES. carmonaedgar@ hotmail.com.
\end{abstract}

***Máster en E-learning. Profesora en la Universidad Central de Colombia. rodrel153@gmail.com.

Cómo citar: Carmona, E.J., Rodríguez, E. (2017).Buenas prácticas en la educación superior virtual a partir de especificaciones de estándares e- Learning. Sophia, 13(1): 13-26.

\section{Resumen}

Actualmente se están ofreciendo cursos libres, programas académicos completos y universidades parcial y totalmente virtuales, lo que evidencia un aumento en la oferta y cobertura en la educación superior. Con el crecimiento surgen nuevas necesidades emanadas de los cuestionamientos sobre la calidad de la educación, que depende de muchos factores, por ejemplo, de la normalización de los espacios académicos on-line o virtuales. En esta investigación se propuso la creación de un conjunto de especificaciones técnicas, administrativas, comunicativas y pedagógicas, para el mejoramiento de la formación virtual en la Universidad del Quindío, que sirva como referencia de buenas prácticas para el docente universitario. En este proceso se recogieron experiencias significativas y, como resultado final, se construyó una propuesta a la luz de las categorías, creadas por organismos internacionales especializados en estándares. Con ello, se iniciaron procesos de normalización, los cuales contribuyen al fortalecimiento de la calidad de la educación.

Palabras clave: Calidad educativa, E-learning, educación virtual, estándares, plataformas educativas.

\section{Abstract}

Free courses, full academic programs, and partially and totally virtual universities are now offered, which shows an increase in the offer and coverage in higher education. With growth, new needs emerge from questions about the quality of education, which depends on many factors; for example, the standardization of online or virtual academic spaces. This research proposed the creation of a set of technical, administrative, communicative and pedagogical specifications to improve virtual training at the University of Quindío, which serves as a reference for good practices for university teachers.

*Artículo resultado del proyecto "Establecimiento de especificaciones para un estándar de calidad e-learning para la Universidad del Quindío", desarrollado por el grupo GEDES. 
In this process, significant experiences were collected and, as a final result, a proposal was built in the light of categories created by international organizations specialized in standards. This led to standardization processes, which contribute to the strengthening of the quality of education.

Key words: Standards, virtual education, educational platforms, E-learning, educational quality.

\title{
Resumo
}

\begin{abstract}
Na atualidade, estão sendo oferecidos cursos livres, programas acadêmicos completos e universidades parcial e totalmente virtuais, o que evidencia um crescimento na oferta e a cobertura do ensino superior. Com este crescimento surgem novas necessidades que emanam das perguntas sobre a qualidade da educação, as quais dependem de muitos fatores, por exemplo, a normalização dos espaços acadêmicos on-line ou virtuais. Nesta pesquisa foi proposta a criação de um conjunto de especificações técnicas, administrativas, comunicativas, e pedagógicas, para o melhoramento da formação virtual na Universidade do Quindío, que servisse como referência de boas práticas para o professor universitário. Neste processo foram recolhidas experiências significativas e como resultado final, foi construída uma proposta de acordo com as categorias criadas por organismos internacionais especializados em padrões. Com isto, foram iniciados processos de normalização, que contribuem ao fortalecimento da qualidade da educação.
\end{abstract}

Palavras- chave: Padrões, educação virtual, plataformas educativas, e-learning, qualidade educativa.

\section{Introducción}

Con la invención del computador a mediados del siglo $\mathrm{XX}$, se resolvieron algunos problemas de la industria, del comercio y de los servicios. Dos aportes tecnológicos significativos, hicieron grandes contribuciones: El computador personal y la aparición de internet. Con ellos se facilitó el acceso de la computación al hogar. Desde entonces se pensó en la posibilidad de utilizar los recursos informáticos en la educación, pero solo al finalizar el siglo XX, se dieron pasos significativos (Barberá, 2008). Es así como surge el concepto de educación virtual, un subconjunto de la educación a distancia, la cual a su vez ha tenido varias etapas, en función de los medios utilizados (Carmona y Berrío, 2008).

Hoy la informática y sus usos han evolucionado a un concepto más amplio: el de las Tecnologías de la Información y la Comunicación (TIC) o para algunos autores Tecnologías de la Información (TI) (Carmona, Nieto y Rubio, 2006), mientras que para otros, Tecnologías de la Información, la Comunicación y el Conocimiento (TICC) (Carrasco \& González, 2011), pero todas estas siglas involucran a un conjunto de tecnologías entre las cuales se incluyen, la informática, telecomunicaciones y redes de dispositivos móviles, luego surgieron las TIC aplicadas a la educación, como sistemas de apoyo, tanto a la formación presencial, como al aprendizaje en línea, creando nuevos entornos, los cuales, oscilan desde la virtualidad como apoyo a la presencialidad, hasta la virtualidad total, en función de los tipos de iniciativa, tecnología utilizada y demanda que se quiere suplir.
La educación virtual en Colombia, es una realidad que se aprecia en la creciente demanda y oferta de programas; a tal punto que todas las universidades ofrecen cursos virtuales (Facundo, 2011, Carrasco \& González, 2011 y Sierra, 2009). Este crecimiento se puede apreciar en la figura 1, donde 2013 existían menos de dos millones de estudiantes presenciales y cerca de 300 mil en distancia y virtual. Para el 2015 el número de estudiantes, sobrepaso los dos millones y los matriculados en modalidad virtual ascendieron a 65.000 , lo que refleja un aumento del $30 \%$ en relación con el año anterior ${ }^{1}$.

La Universidad del Quindío también ha ido aumentando paulatinamente la oferta de cursos virtuales. Pero en este crecimiento no ha mediado un proceso de planeación sistemático y ordenado. Se hace necesario entonces emprender varias acciones, que orienten no sólo la implementación de cursos y programas virtuales, sino además, el mejoramiento de la educación, utilizando las potencialidades de las TIC. La normalización y estandarización es una buena alternativa para iniciar procesos que conduzcan a garantizar programas virtuales de alta calidad, en atención a que uno de los factores más importantes para que cualquier tipo de material instructivo sea de calidad, es que sea útil para el logro de aprendizajes, bajo normas y estándares mínimos.

1. De acuerdo con Portafolio, http://www.portafolio.co/economia/ finanzas/hay-65-000-estudiantes-virtuales-colombia-22356. 
Donna Zapata en un documento realizado por el Instituto Colombiano para el Fomento de la Educación Superior (ICFES) ${ }^{2}$, afirma que si Colombia quiere aprovechar los adelantos de las TIC a favor de la educación, necesita agilizar los proyectos de conformación de redes universitarias que se vienen gestando desde hace algún tiempo. A manera de conclusión, afirma que el trabajo de las instituciones de Educación Superior, no es suficiente para responder a las demandas de modernización y calidad que se requieren para que la educación pueda constituirse en agente de progreso para el país (Zapata, 2003).

Figura 1. Número de estudiantes matriculados en educación superior en Colombia.

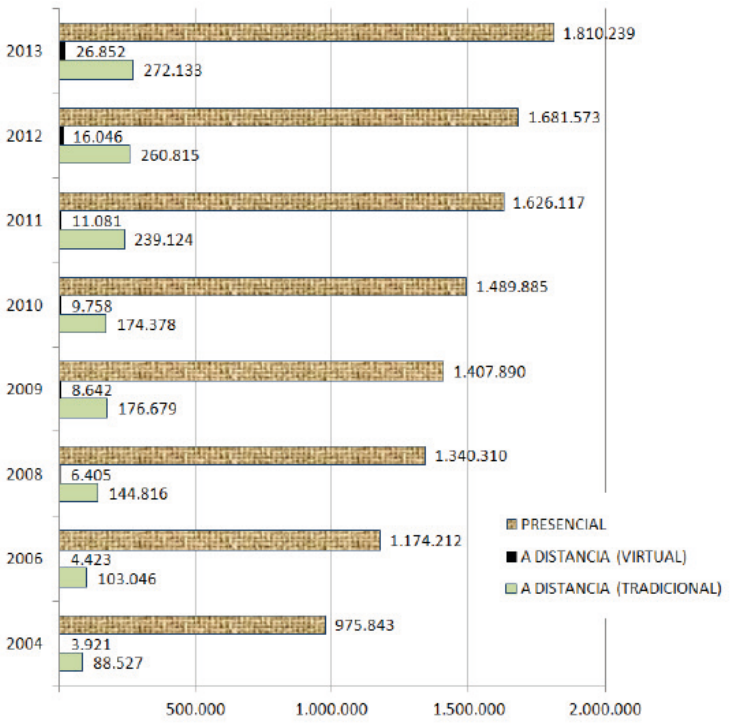

Fuente: Sistema Nacional de información la Educación Superior (SNIES) - Ministerio de Educación Nacional.

Las universidades - incluidas las más tradicionalistas - ya ofrecen cursos virtuales (Facundo, 2011, Rodríguez \& Sierra, 2007 y Aldana, 2003). A manera de ejemplo, la Universidad Central de Colombia dispone de cursos de extensión y programas de postrado en modalidad virtual, atendiendo las directrices impulsadas por el Ministerio de Educación Nacional (MEN), relacionadas con la modernización e innovación en el sector. En dicho contexto la Universidad ha consolidado su propuesta virtual, bajo un modelo de formación de excelencia, basado en los estándares de calidad académica. En el 2013 el MEN

2 Estudio realizado por el ICFES organismo rector de la educación superior en Colombia. concedió a la Universidad Central el primer Registro Calificado al Programa de Postgrado en Mecatrónica Industrial, $100 \%$ virtual.

Para el caso de la Universidad del Quindío, en el marco de este proyecto, se hizo un análisis de la forma cómo se han implementado la educación virtual, mediante entrevistas a los principales actores, revisión documental y conversaciones directas con los profesores. Se destaca cómo desde el año 2000 cuando se iniciaron los primeros esfuerzos por iniciativa de unos docentes, hasta el momento se ha conformado una dependencia que centraliza todos los procesos de virtualidad, denominada la Unidad de virtualización (UdV).

La Unidad de Virtualización nace en 2008, como evolución del Proyecto Aula Virtual de la Facultad de Educación. En ese momento los lineamientos del MINTIC, se orientaban al diseño de programas virtuales, fue así como la Universidad desarrolló un currículo para los ciclos técnico y tecnológico en el área de Turismo (ver figura 2).

Posteriormente, se diseña el diplomado en Ambientes Virtuales de Aprendizaje, el cual tiene como propósito: formar a los docentes de la Universidad, para el desempeño en ambientes de aprendizaje mediados por plataformas LMS. De este diplomado se han graduado 9 cohortes.

Figura 2. Acciones en la Unidad de Virtualización en Uniquindío

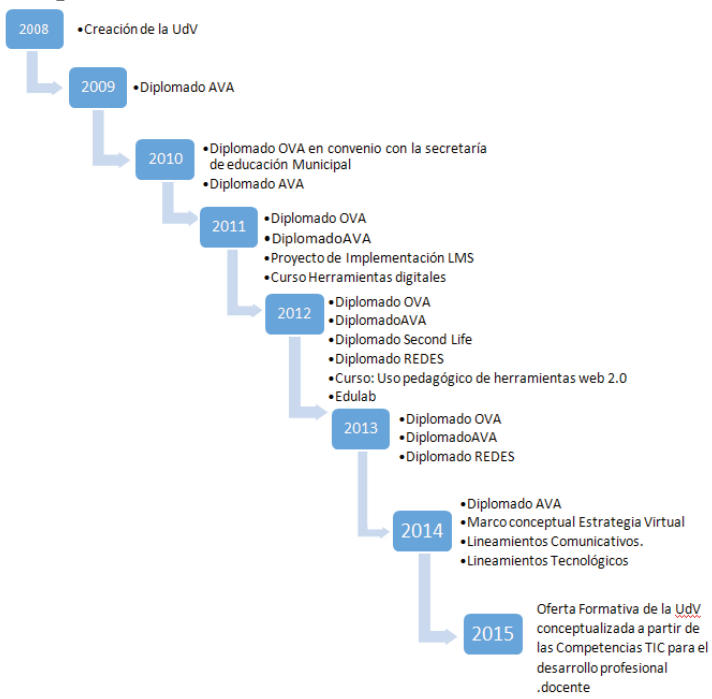

Fuente: Unidad de virtualización y elaboración propia. 
Bajo los mismos lineamientos, se diseña el diplomado en Objetos Virtuales de Aprendizaje (OVA), el cual se transforma en Diplomado en Recursos Educativos Digitales Abiertos (REDA) - a partir del primer semestre del 2015 - debido a los replanteamientos conceptuales del MEN y siguiendo la filosofía de los Open Educational Resources. Los esfuerzos de la Unidad de Virtualización se concentran entonces en:

a) Sensibilizar a los docentes sobre la importancia de implementar tecnologías dentro de sus diseños pedagógicos.

b) Estructurar un equipo profesional que responda a las complejidades inherentes a la implementación tecnológica, desarrollando estrategias en cuatro componentes:

Componente organizacional: en el que se desarrollan las gestiones necesarias para permitir el funcionamiento de la Unidad de Virtualización.

Componente pedagógico: acompaña los diseños curriculares de los docentes.

Componente tecnológico: se concentra en el mantenimiento y administración de la plataforma Learning Managment System (LMS).

Componente comunicativo: se ocupa de establecer lineamientos para el diseño y la producción de contenidos educativos, incorporando diversos medios y TIC, nuevas formas narrativas y tipologías textuales.

La Unidad de Virtualización se encarga de administrar las plataformas:

Espacios académicos virtuales: aloja los cursos que son proyectos elaborados por los docentes formados en el diplomado AVA (cuenta con 40 cursos).

Plataforma Recursos de Apoyo: alojan los cursos producidos por profesores autodidactas que crean recursos, los cuales son el resultado de sus ejercicios personales orientados a explorar las herramientas de la plataforma; estos cursos por lo general no cumplen con los lineamientos establecidos por la Unidad de Virtualización (Cuenta con 168 cursos).

Plataforma de pruebas: en esta plataforma se hacen las diferentes pruebas de implementación de nuevas funciones de Moodle.
Además de estas plataformas, el Campus Virtual de la Universidad cuenta con una instalación adicional de Moodle para atender todas las asignaturas del programa de Ciencias de la Información, Documentación, Bibliotecología y Archivística (CIDBA), que se ofrece completamente virtual.

El equipo de trabajo de la UdV ha visto la necesidad de establecer orientaciones para la estrategia virtual, entendiendo que es una acción transversal dentro de la estructura organizacional de la Universidad del Quindío.

En este orden de ideas, se ve la necesidad de construir diferentes documentos para socializar los elementos conceptuales -organizacionales, pedagógicos, comunicativos, tecnológicos- constitutivos de la estrategia virtual, con el propósito de unificar criterios dentro de la comunidad académica. Estos documentos son:

- Marco conceptual.

- Infografía conceptos estrategia virtual.

- Lineamientos pedagógicos.

- Infografía lineamientos pedagógicos.

- Lineamientos comunicativos.

- Infografía lineamientos comunicativos.

Así mismo, la UdV lleva a cabo actividades de gestión al interior de la institución educativa con el propósito de promover la apropiación de la estrategia virtual por cada uno de los programas académicos. Para ello, hace convocatoria permanente en los temas relacionados con la formación de competencias TIC para el desarrollo profesional docente.

Sin embargo, en la Universidad del Quindío como en la gran mayoría de las instituciones, y tal como lo afirma Hilera y Hoya, toda esta proliferación de cursos virtuales surgieron y se desarrollaron sin un marco adecuado en cuanto a metodologías técnicas, documentales y psicopedagógicas, lo que conduce a problemas de accesibilidad, interoperabilidad, durabilidad y reutilización de los materiales curriculares, disponibles en las diferentes redes de comunicación (Hilera González \& Hoya Marín, 2010). Se precisa entonces, la utilización de procesos de normalización, que orienten una mejor calidad de los materiales producidos y, por consiguiente, de la calidad de la formación. Esto conduce al concepto de "estándar". 
Según la International Organization for Standarization (ISO), que reúne las diferentes organizaciones nacionales de estandarización: La estandarización es la actividad que tiene por objeto establecer, ante problemas reales o potenciales, disposiciones destinadas a usos comunes y repetidos, con el fin de obtener un nivel de ordenamiento óptimo en un contexto dado, que puede ser tecnológico, político o económico. (ISO, 2009).

Existen dos tipos de estándares: los oficiales o "de jure" y los "de facto". Los primeros, son aquellos que han sido aprobados y sancionados por un organismo nacional o internacional de estandarización. Estos, en algunos casos, son de obligado cumplimiento, por ejemplo: que todas las páginas web oficiales deban cumplir un determinado nivel de accesibilidad para discapacitados. Los estándares de facto son los que se usan por voluntad propia o conveniencia y tienen una amplia aceptación, aunque no hayan sido sancionados por un organismo de estandarización. El caso más conocido en internet, son las recomendaciones realizadas por el World Wide Web Consortium (W3C), que crea las normas probablemente más utilizadas en la red, por ejemplo: el lenguaje HTML (y que en muchos casos, después de publicadas, pasan a ser reconocidas como estándares formales).

Los estándares e-learning representan la posibilidad de evitar diversos problemas originados en la práctica pedagógica mediada por las TIC, entre ellos: el de portabilidad, es decir, que los contenidos se puedan mover y almacenar en diferentes plataformas; la interoperabilidad, esto es, que funcionen en distintas plataformas de hardware y software, sean reutilizables, sirvan como base para la creación de otros cursos y aplicaciones, sean accesibles y usables, que tengan metadatos para facilitar su localización. Hasta el momento, no existe una metodología única que garantice los objetivos de accesibilidad, interoperatividad, usabilidad, accesibilidad y reutilización de los materiales educativos en la red (Hilera \& Hoya, 2010;Wiley, 2007).

Varias organizaciones han generado estándares e-learning con el propósito de sistematizar los procesos, crear materiales educativos compartidos y de calidad. Entre ellas se pueden citar: ADL (La Advanced Distributed Learning), AICC (Aviation Industry Computer Based Training Comitte o Comité de la Industria de la Aviación para el aprendizaje basado en computadoras), CEN (Comité Europeo de Normalización), IEEE (Institute of Electrical and
Electronic Engineers, y dentro de él, el LTSC o Comité de Estandarización de las Tecnologías Aplicadas al Aprendizaje), IMS GLC (IMS Global Learning Consortium), ISO/IEC (International Standards Organisation en colaboración con International Electrotechnical Commission) ARIADNE (Alliance of Remote Instructiona Authoring and distribution Network for Europe), DCMI (Dublin Core Metadata Intiative) y W3C (World Wide Web Consortium).

Estas organizaciones han creado estándares que se encargan de normalizar el E-learning categorizado en: contenidos y evaluaciones, usabilidad, accesibilidad, interoperatividad, arquitectura, calidad, competencias, derechos de autor, información del estudiante, metadatos, procesos de aprendizaje, repositorios, vocabulario y lenguajes. Algunos de ellos son: AGR009 (1996), EFQM (1999), AGR002 (2002), IEEE 1482.2 (PAPI) (2002), CanCore ES (2002), EdNA MD (2002), UNE 139801 (2003), IMS ACCMD (2004), IMS DALA (2005), CORDRA (2005), SCORM CAM (2006), UNIQUe (2006), CWA 15533 (2006), HR-XML (2007), ISO/IEC 24751-1 (2008), ISO 9001 (2008), LORN MD (2008) y WAI ARIA (2009).

En Colombia en 2005 el Ministerio de Educación Nacional (MEN), lanzó el Primer Concurso Nacional de Objetos de Aprendizaje, con el propósito de consolidar, por primera vez, un banco de objetos de aprendizaje y ponerlo a disposición de la comunidad educativa nacional e internacional, uno de los requerimientos que se hizo a los participantes, fue la creación de una ficha técnica con todos los atributos y descripción de uso del objeto de aprendizaje, de tal forma, que permitiera una fácil catalogación e intercambio de los mismos. Lo anterior, obligó a pensar en la búsqueda, análisis y revisión de estándares existentes a la fecha, de este estudio, el MEN adoptó el metadato Dublin Core, el empaquetamiento mediante Scorm, el sistema de administración de contenidos (CMS) Drupal y DSpace para conformar el Banco de Objetos ( MEN, 2012).

Un año más tarde fue necesario hacer visible y utilizables, los objetos de aprendizaje creados, para lo cual, se pensó en un estándar de clasificación fácil y oportuna, para el acceso a los objetos, los cuales estuvieran dispuestos en categorías y áreas del conocimiento, por lo cual, se definió entonces un perfil de aplicación de un estándar de metadatos, que se basó en el estándar IEEE LOM y ajustando el perfil de aplicación a los requerimientos propios 
del país, ha implementado una adaptación de este estándar que ha denominado LOM CO, para describir los aspectos técnicos y educativos de los objetos del Banco Nacional y de los bancos institucionales.

LOM CO define los siguientes metadatos obligatorios para los Objetos de Aprendizaje del Banco Nacional y considera opcionales, los correspondientes al resto de campos de la especificación IEEE LOM (Agudelo Benjumea, 2009):

a) General: título, idioma, descripción, palabras clave.

b) Ciclo de vida: versión, autor(es), entidad, fecha.

c) Técnico: formato, tamaño, ubicación, requerimientos, instrucciones de uso.

d) Educacional: tipo de interactividad, tipo de recurso de aprendizaje, nivel de interactividad, población objeto, contexto de aprendizaje.

e) Derechos: costo, derechos de autor y otras restricciones.

f) Relación: define la relación entre objetos de aprendizaje.

g) Anotación: uso educativo.

h) Clasificación: fuente de clasificación y ruta taxonómica.

El Gobierno colombiano tomó conciencia acerca de la importancia de la educación virtual y por ello promulgó el Decreto 1295 de 2010, por medio del cual dotó a la educación de herramientas que desarrollaran una nueva filosofía educacional, y es hacia allá donde debe apuntar la tarea del docente, de entender que sin esta no podemos tener altos estándares de calidad, suficientes para entregar profesionales probos al sector laboral del país (Zuluaga, 2010).

Con lo anterior, las condiciones para la investigación estaban dadas. De un lado, una tendencia mundial, y de otro, algunos avances a nivel nacional. Se justifica esta investigación, por cuanto es importante determinar para nuestra institución, los lineamientos en materia de estandarización de la educación virtual.

El objetivo principal de esta investigación es formular un conjunto de especificaciones que orienten la calidad de la educación virtual desde el establecimiento de un estándar e-learning, de manera que facilite la normalización de los procesos en la modalidad virtual en la Universidad del Quindío. Para ello, se debía cumplir con las metas intermedias como: a) Hacer un diagnóstico de la forma como se han implementado procesos de educación virtual en la Universidad del Quindío.

b) Analizar los diferentes procesos de normalización y estandarización para la educación virtual, que a nivel mundial se han venido generando, y particularmente, los que adoptó el Ministerio de Educación Nacional de Colombia.

c) Elaborar un conjunto de especificaciones técnicas, administrativas y pedagógicas del e-learning para la Universidad del Quindío, en el que se proponga un plan de acción que contribuya a la implementación de las normas que orienten a la calidad educativa.

\section{Materiales y métodos}

La investigación tiene una orientación cualitativa, ya que combina técnicas etnográficas y de Investigación Acción Participación (IAP). El componente etnográfico se fundamenta en la reflexión en torno al estudio de la evolución de la virtualidad en la Universidad, al análisis del estado del arte de los estándares en educación virtual y a la forma como los artefactos tecnológicos median dinámicas educativas. Desde la perspectiva de la IAP se elabora una estrategia de mejoramiento, mediante la creación de un conjunto de especificaciones que se materializan en un manual de buenas prácticas y en un diseño metodológico para la implementación de dicho manual.

Se hizo inicialmente una revisión bibliográfica del estado del arte en estándares para educación virtual lo que permitió la fundamentación teórica; luego, una observación activa, mediante la visita a cinco universidades, con el fin de hacer una contextualización nacional sobre el estado del arte. Al interior de la Universidad del Quindío, se realizaron entrevistas no estructuradas a líderes de estrategias virtuales y encuestas a docentes orientadores de cursos virtuales. Mediante instrumentos de observación a una muestra de los cursos en funcionamiento en la plataforma educativa se realizó el análisis del estado del e-Learning. La población objeto de estudio está conformada por cuarenta docentes que participan en la formación virtual, a quienes se les aplicaron los instrumentos y métodos de observación planteados. 


\section{Resultados}

Se partió del estudio de la implementación de procesos virtuales en la educación superior y particularmente en conceptos relacionados con los estándares de calidad, es decir, las características deseables para generar ambientes virtuales exitosos.

Después análisar los diferentes estándares y especificaciones utilizados en la enseñanaza, y mundialmente reconocidos por los organismos citados, se encontró una amplia gama de propuestas, lo que limita el análisis de algunos estándares. De igual manera, se detectó que ningún estandar cubre todos los aspectos que un contexto universitario demanda. Aquí coincidimos con el informe 16 del Ministerio de Educación y Ciencia de España, en el cual se les denomina como "los más maduros" y son aquellos que abordan aspectos relativos a los contenidos, cómo se empaquetan los cursos, cómo se describen tanto los cursos como los propios elementos que los componen (Fernández, Moreno, Sierra, \& Martínez, 2008).

Se consideró en concordancia con Adell, Bellver y Bellver (2007), que tres experiencias son suficientemente argumentadas, como para ser consideradas en procesos de estandarización en cualquier institucion universitaria, son ellas: IMS (Global Learning Consortium, Inc.) es el principal promotor y desarrollador de especificaciones abiertas en aspectos de la educacion virtual; el desarrollado por ADL en su modelo de referencia Scorm, y, el aporte de IEEE LTSC con su propuesta de metadatos para objetos de aprendizaje.

En el ámbito local se observó que la Universidad del Quindío contempla como uno de sus ejes estratégicos para la excelencia académica, la adquisición de instrumentos didácticos, tecnología y medios informáticos, en concordancia con los lineamientos del Ministerio de Educación Nacional. Para el cumplimiento de este propósito, se hace un análisis del estado del arte, con respecto a las nuevas formas de aprendizaje, algunas tendencias en la sociedad del conocimiento y cómo utilizar nuevos instrumentos para facilitar el proceso de enseñanza-aprendizaje. De allí surge el siguiente interrogante: ¿Los estándares y lineamientos que permiten la construcción de proyectos educativos tecnológicos, innovadores y creativos contribuyen a la calidad educativa? En el intento por responder a esta pregunta, los investigadores empiezan a encontrar claridad con respecto a estrategias de implementación tecnológica, que impacten positivamente los espacios de aprendizaje al interior de la Universidad.

Finalmente, se obtuvo un instrumento para la acción, es decir, un documento que sirve de guía a las instituciones para el desarrollo futuro en educación virtual y al docente para que pueda, de manera más asertiva, enfrentarse a procesos de virtualización. Se consideró que tiene un componente de investigación-acción, así como la condición que se tiene de investigadores, inmersos en el sistema objeto de estudio.

\section{Discusión de resultados}

Si bien los estándares están construidos para cumplir normas, en este ejercicio de reflexión, diseño e investigación, se ha comprendido la necesidad de acomodar a situaciones contextuales y especificaciones generalizantes, entendiendo que en la compleja realidad educativa, interactúan variedad de factores que estimulan o interfieren los procesos de aprendizaje. Es claro que el seguimiento de una política internacional en esta materia, si bien permitiría a la Universidad, una normalización mundialmente reconocida y avalada, de otro lado, es importante considerar que una amenaza por superar, es responder a las necesidades particulares. En los siguientes apartados se describe la propuesta realizada, para dar cumplimiento a los objetivos propuestos.

\section{Propuesta de especificaciones para los estándares de educación virtual}

Varios autores coinciden en la necesidad de considerar las categorías que fundamentan los requerimientos del estándar (Viscay, 2005, Hilera González \& Hoya Marin, 2010). Para el caso de la Universidad del Quindío se consideró importante la definición de las siguientes características:

Transversalidad: los contenidos educativos que traten temas generales y puedan ser usados por diferentes áreas del conocimiento (Ética, Emprendimiento, proficiencias, Constitución Política, etc.).

Transmedia: los contenidos educativos deberán prepararse, diseñarse y producirse, aprovechando la peculiaridad del medio, dentro de esta característica se apela a la granularidad, que hace referencia al 
aprovechamiento narrativo del medio que lo soporta y, da la posibilidad de articularse a estructuras globales (cursos).

Diseño funcional: cada contenido educativo debe responder a un propósito y a una intención educativa. Los docentes han de trabajar de manera articulada, con equipos creativos, expertos en diseño, formas narrativas, animación y composición, para orientar de la mejor manera la producción de los contenidos educativos.

Gestión administrativa: cada contenido educativo debe responder a una necesidad académica que fundamente, soporte $\mathrm{y}$ oriente el proceso de producción.

Licenciamiento: establecer términos y condiciones claras para el uso, modificación y personalización de los contenidos educativos, y, en lo posible, la promoción del copyleft, a través de las licencias Creative Commons, y particularmente, las Creative Commons Colombia, sin desconocer los sistemas tradicionales de licenciamiento.

Unidad conceptual: la institución debe establecer un marco conceptual para el entendimiento y difusión del proyecto educativo institucional.

Igualmente, para presentar la propuesta de estándar para los cursos mediados con tecnología, se consideró importante y fundamental retomar los conceptos establecidos en el marco conceptual de la Estrategia Virtual de la Universidad del Quindío, los cuales definen las diferentes metodologías, dentro de las que se desarrollan cursos e-learning y b-learning, tal como se aprecia a continuación:

Presencial: Se caracteriza por la realización de "clases magistrales", con apoyo de unidades didácticas y herramientas como proyectores, tableros o pizarras interactivas (táctiles).

Virtual - e-learning: Metodología que se lleva a cabo a través del uso de medios y tecnologías de la información y la comunicación - MTIC. Incorpora diseño pedagógico y metodológico en el desarrollo de los contenidos temáticos y contempla actividades $100 \%$ virtuales entre los participantes (profesor/estudiantes). Generalmente el desarrollo de actividades se realiza de manera asíncrona.
B-learning: Combina las metodologías virtual y presencial. Incorpora el diseño pedagógico y metodológico en el desarrollo de los contenidos temáticos, distribuidos con una proporción mínima del $60 \%$ para actividades virtuales y el $40 \%$ para actividades presenciales.

Distancia: Según el decreto 1295 de 2010, "corresponde a aquellos cuya metodología educativa se caracteriza por usar estrategias de enseñanza aprendizaje que permite superar las limitaciones de espacio y tiempo entre los actores del proceso educativo".

\section{Concepto de estándar para los cursos b-learning y e-learning}

De acuerdo con los organismos internacionales y en consideración con los aspectos particulares para el caso de la Universidad, se elaboró una definición. Se concibe entonces un estándar como: un conjunto de especificaciones técnicas, pedagógicas y administrativas que regulan el diseño, la producción y la implementación de los recursos educativos, cuyo objetivo es la interoperabilidad entre dichos recursos construidos por diferentes profesores, en diferentes programas, con variedad de soportes y bajo criterios de unificación, normalización y racionalidad del costo.

Dichas especificaciones poseen características abiertas que propician la construcción colaborativa de recursos educativos, entendidos estos en una acepción amplia que comprende la dimensión más granular de un recurso educativo: los Objetos de Aprendizaje, hasta la estructura más compleja: las plataformas LMS.

\section{Especificaciones para el estándar}

Se presenta para esta investigación, un conjunto de especificaciones, resultantes de la revisión documental internacional y de la conceptualización de acuerdo con particularidades detectadas en la realidad educativa como en el contexto local. Los criterios de respeto por los derechos de autor, así como los aspectos administrativos, son algunos de los componentes importantes a la hora de implementar estrategias tecnológicas dentro del Proyecto Educativo Institucional (PEI) de la Universidad del Quindío. 
Todos los estándares están clasificados en categorías, las cuales a manera de fragmentación del problema crean especificaciones. Estas especificaciones son: usabilidad, accesibilidad, adaptabilidad, modularidad, didáctico, pedagógico, comunicativo, clasificable y licenciado. A continuación, se presentan un conjunto de especificaciones que componen, según esta propuesta, el estándar de calidad e-learning y b-learning para la Universidad del Quindío. Dichas especificaciones han de tenerse en cuenta al momento de diseñar y planear recursos educativos.

\section{Especificaciones para un estándar}

\section{Accesibilidad $^{* 3}$ :}

Cualidad que busca garantizar que el curso o recurso pueda ser consultado y/o utilizado por el mayor número de personas, incluyendo a quienes se encuentran en condición de discapacidad, y de igual forma, a aquellos que no cuentan con condiciones técnicas y tecnológicas adecuadas.

El curso tendrá una versión of-line disponible en soporte portable (CD- USB).

Los recursos educativos del curso deben estar licenciados de manera tal, que permitan su acceso y distribución a través de soportes como CD- USB.

\section{Adaptabilidad}

Propiedad que le permite a los recursos: ser modificados, ajustados o personalizados de acuerdo con los intereses, necesidades o expectativas del usuario.

\section{Usabilidad}

Propiedad que garantiza la correcta interacción con el usuario, con el fin de procurar una experiencia cómoda, agradable, fácil y eficiente.

\section{Modularidad}

Capacidad que permite interactuar o integrarse con otros, en igual o diferentes condiciones y contextos, y con ello ampliar sus posibilidades de uso educativo. El diseño del curso debe considerar cada actividad de aprendizaje como una unidad capaz de combinarse y recombinarse de acuerdo con las necesidades del profesor y de la asignatura. Las actividades deben ser susceptibles de ser compartidas por el grupo docente que orienta la asignatura.

\section{Pedagógico}

Característica que incorpora una función formativa, abarca desde la estructura más granular - un objeto de aprendizaje - hasta la más compleja -una plataforma-. Las actividades de aprendizaje deben responder a las competencias que el estudiante debe desarrollar. Dichas competencias orientan los propósitos educativos de las actividades de aprendizaje.

\section{Comunicativo}

Propiedad que garantiza la claridad del mensaje en lo relacionado al uso de los recursos y al contendido en sí mismo.

El curso debe presentar procedimientos para el desarrollo de las actividades de aprendizaje y mediaciones necesarias para comprender el manejo de aplicaciones digitales y equipos de laboratorio y práctica, estos recursos deben estar dispuestos dentro de la actividad de aprendizaje.

\section{Clasificable}

Propiedad que posibilita su catalogación y ordenamiento.

\section{Licenciado}

Característica que define las condiciones de uso de los recursos.

\section{Componentes de un curso desde la perspectiva del estándar}

Tal como se observa en la figura 3 , un curso mediado con tecnologías es el resultado de la articulación de diferentes componentes, entendidos estos como recursos tecnológicos y procedimientos y gestiones necesarias para su viabilización.

3. as definiciones de las especificaciones marcadas con asterisco son tomadas de las características de los Recursos Educativos Digitales Abiertos (REDA), definidos en el Documento REDA del MEN. 
Figura 3. Componentes de un curso.

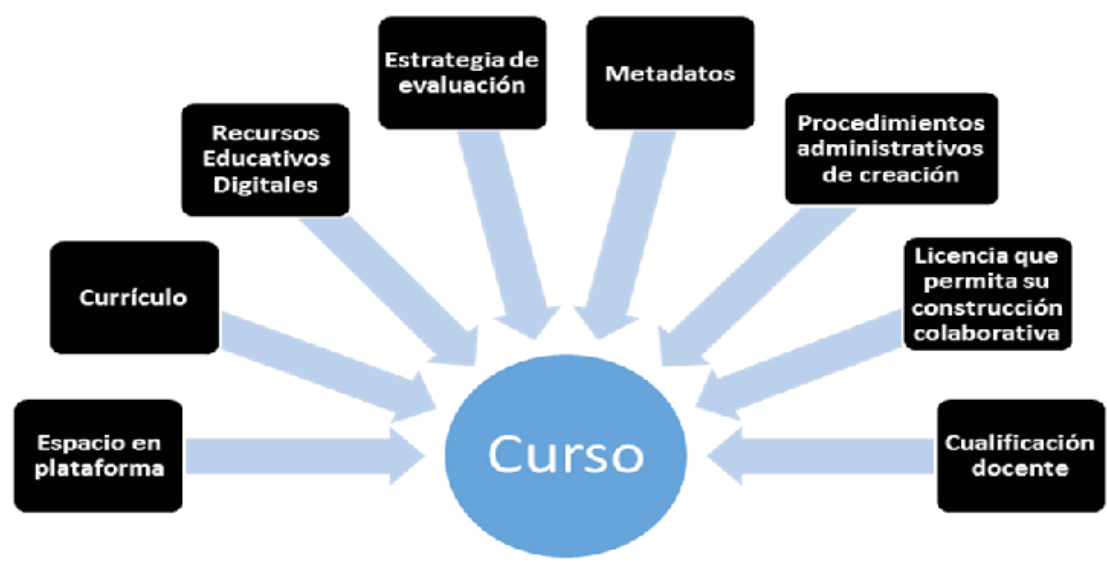

Fuente: elaboración propia

\section{Aplicación de especificaciones}

Para la implementación de dichas especificaciones se tienen en cuenta documentos y procedimientos ya definidos dentro de la Estrategia Virtual de la Universidad del Quindío. Estos insumos son utilizados en el flujograma de la figura 4, que representa el procedimiento para la creación de cursos mediados con tecnología.

Figura 4. Procedimiento de creación de cursos.

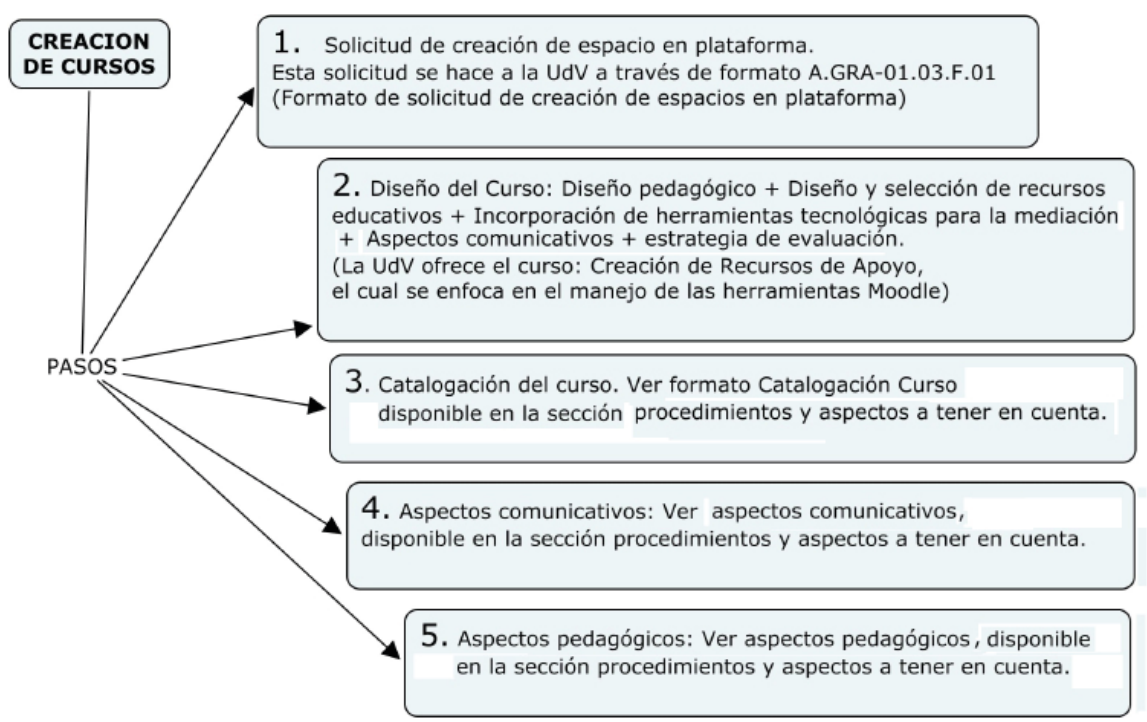

Fuente: Unidad de virtualización y elaboración propia 


\section{Configuración general de los cursos}

Apartir de la implementación de estas especificaciones, los cursos que se crean en las plataformas, deberán seguir las recomendaciones para la configuración general del curso, tal como se observa:

\section{Recomendaciones para la configuración de cursos}

\section{Nombre del curso o espacio formativo}

Debe reflejar el contenido general del curso, este debe ser claro y conciso.

Debe contener mayúscula inicial en cada palabra, excepto en las preposiciones, las siglas deben estar en mayúsculas. Ejemplo: Estrategias para Desempeño en AVA Cohorte IX G.

\section{Nombre corto}

Debe contener: Las iniciales de la facultad, año, semestre (entendido este como el período académico del año) y las iniciales del curso.

Las iniciales de la facultad serán las siguientes:

- Ciencias Humanas y Bellas Artes: $\quad \mathrm{CH}$

- Educación:

- Ciencias de la Salud:

CS

- Ingeniería:

IN

- Ciencias Económicas y Administrativas: CE

- Ciencias Agroindustriales:

- Ciencias Básicas y Tecnológicas:

Del año se tomarán los 2 últimos dígitos.

El semestre o período académico del año y se codificará como 1 y 2 .

Ejemplo: el curso CH142CAG3, significa que es de la Facultad de Ciencias Humanas, año 2014, segundo semestre - Comunicación Asertiva y es el grupo 3.

\section{Programa académico}

Nombre del programa académico o dependencia administrativa a la cual pertenece el curso o espacio formativo.

\section{Descripción general del curso}

Descripción del curso en 140 caracteres. El texto debe contener una síntesis sobre los aspectos fundamentales del curso, tales como: temas, competencias a desarrollar (solo mayúscula inicial).

\section{Número de unidades}

Se describe el número total de unidades, módulos y secciones que conforman el curso.

\section{Aspectos comunicativos}

La comunicación se considera un componente de gran importancia en la calidad del curso, por ello se considera que todo curso debe considerar los siguientes aspectos:

\section{Presentación del docente}

El curso debe contener un video de presentación del docente, quien rápidamente hace una presentación personal y profesional. Este video puede estar integrado con el video de presentación del curso.

\section{Presentación del curso}

Esta presentación puede hacerse a través de video o de audio. En este recurso, el docente hace una síntesis o introducción que permita al estudiante conocer las generalidades del curso (contenidos. evaluación, metodología, horarios, cronograma, etc.).

\section{Medios de asesoría docente}

El docente debe explicitar los medios a través de los cuales hará asesorías y seguimiento a los estudiantes, los horarios de atención y lugares de encuentro.

\section{Tutoriales y videotutoriales}

Si se requiere el docente debe facilitar las mediaciones necesarias para que los estudiantes conozcan cómo utilizar equipos y aparatos dentro de las diferentes prácticas y laboratorios.

\section{Tiempo de respuesta}

El tiempo de respuesta a las consultas e inquietudes de los estudiantes del curso ha de darse en un tiempo no mayor a 24 horas. 


\section{Foro dudas e inquietudes}

Este espacio de participación le ofrece la oportunidad de consignar las inquietudes de interés común sobre los contenidos y actividades del módulo. Este foro debe proponerse como un espacio colaborativo, donde se encuentran respuestas y orientaciones, no solo por parte del profesor, sino también de los demás compañeros integrantes del grupo.

\section{Foro conozcámonos (Novedades)}

Este espacio permitirá a los estudiantes, conocerse e interactuar con sus compañeros de curso.

\section{Perfil}

El perfil debe contener datos actualizados tanto del estudiante como del docente. Datos de contacto - fotografía - pregrado que estudia - intereses: académicos, culturales y deportivos

Para los docentes, deberá contener además, la formación profesional y experiencia laboral.

\section{Calendario}

Este recurso relaciona el desarrollo de las actividades del curso, mostrándolas en el bloque de eventos próximos.

\section{Aspectos pedagógicos}

El componente más complejo, dada la magnitud, en la creación de cursos es el uso contundente de argumentos pedagógicos que contribuyan al logro de los objetivos trazados de manera eficiente, pues depende de muchos factores e involucra en gran medida las actividades del docente en el desarrollo día a día de los cursos. Un curso estratégicamente diseñado, con un componente pedagógico definido, se espera que sea un curso exitoso, pero siempre está en función del profesor que oriente el curso. Sin embargo, se proponen como aporte al componente pedagógico, las siguientes especificaciones:

\section{Guía general}

Como parte del desarrollo del curso virtual es necesario que el docente elabore una guía general del curso disponible en plataforma.

\section{Metodología}

Teniendo en cuenta los créditos académicos o la intensidad horaria del curso virtual, así como las relaciones entre tiempo de estudio independiente del estudiante (TI), el tiempo para el trabajo colaborativo entre estudiantes (TC) y el tiempo de trabajo de tutoría o acompañamiento por parte del docente-tutor (TT), el docente debe proponer un cronograma de trabajo que le permita al estudiante, organizar sus tiempos de estudio diario o semanal, especificando cuáles de las actividades de aprendizaje propuestas serán retroalimentadas y calificadas, así como su porcentaje frente a la calificación final.

Esto implica prever con especial cuidado, cuantificar el tiempo que debe dedicar el estudiante al estudio del material de lecturas, al abordaje de actividades, a la búsqueda de información o trabajo en equipo, preparación de los aportes académicos, y demás desempeños en que deberá incurrir el estudiante bajo los principios de autodisciplina y aprendizaje colaborativo, precisando también los tiempos en los que acompañará o recibirá retroalimentación del tutor.

\section{Red conceptual}

Muestra un esquema o mapa de conceptos propios del curso.

\section{Producción intelectual propia}

Se debe priorizar la elaboración de contenidos propios que expresen las ideas, pensamientos y criterios de los docentes, frente a otras fuentes y otros autores. Esto con miras a ampliar el repositorio de recursos educativos disponibles para el desarrollo de las actividades de aprendizaje.

\section{Evaluación}

Se deben acoger las recomendaciones sobre la evaluación del aprendizaje, adoptada por la Universidad en el respectivo sílabo. Para el diseño, montaje y desarrollo de cursos, se debe identificar el desplazamiento que va desde la calificación a la evaluación, y esta se deberá especificar de manera cualitativa hasta la formulación de la forma cómo el trabajo del participante será evaluado.

Además se deben especificar los porcentajes de cada uno de los componentes de la evaluación. 
En los foros se evaluará la calidad de las intervenciones allí consignadas.

\section{Recursos educativos}

Todos los libros, lecturas, recursos y material complementario para el logro de los objetivos deben ser facilitados, respetando los derechos de autor.

\section{Bibliografía / webgrafía}

El docente debe aportar todos los link o enlaces de acceso al material de estudio: sitios, bibliotecas digitales o repositorios de contenidos educativos, teniendo en cuenta derechos de autor y licencias de uso.

Lo anterior sintetiza los aspectos comunicativos, pedagógicos y la configuración de los cursos, que unido a procedimientos para su creación, conforman el conjunto de especificaciones para orientar los procesos e-Learning, con la expectativa que se conviertan en un estándar para la Universidad del Quindío.

\section{Conclusiones}

Como un primer resultado de la conceptualización y revisión documental de los estándares internacionales, se hizo una caracterización de los procesos virtuales en la Universidad del Quindío y su ubicación en el contexto global. De allí surgió la importancia de considerar componentes esenciales a la hora de implementar estrategias de educación virtual, tales como: el respeto por los derechos de autor, aspectos técnicos y académico-administrativos.

Se reconoce la condición virtual como otra forma de participación de la Universidad en la comunidad regional, que demanda el diseño de estrategias con el objeto de ofrecer procesos educativos de calidad que sean útiles en las diferentes modalidades (presencial - distancia - virtual).

La usabilidad, accesibilidad, adaptabilidad y modularidad, son conceptos validados y compartidos a nivel internacional, presentados por diferentes entes especializados en estandarización. En consonancia, transversalidad, diseño funcional, gestión administrativa, licenciamiento y unidad conceptual, son conceptos que responden a reflexiones en torno a las peculiaridades educativas y contextuales.
Un ambiente de aprendizaje es efectivo si tiene coherencia entre los lineamientos que fundamentan su diseño: pedagógicos, comunicativos, organizacionales y tecnológicos. Si bien los estándares están construidos para cumplir normas, en este ejercicio de reflexión e investigación, se ha comprendido la necesidad de acomodar a situaciones contextuales, especificaciones generalizantes. Entendiendo que la compleja realidad educativa debe ser abordada desde un pensamiento flexible, los estándares se conciben como especificaciones que orientan el diseño de procesos educativos que incorporan lo virtual y lo tecnológico.

Debido a que las prácticas educativas están fuertemente mediadas por artefactos digitales, estos deben ser pensados y diseñados desde perspectivas que posibiliten que el conocimiento sea distribuido y compartido; en este orden de ideas la incorporación de estándares en educación debe estar orientada a dar claridad en los procedimientos a tener en cuenta tanto en la producción como en la implementación de las mediaciones tecnológicas. La normalización debe aplicarse con el propósito de establecer procesos y procedimientos que articulen y hagan evidente en cada diseño el diálogo coherente de los lineamientos que constituyen la Estrategia Virtual.

Se diseñó un conjunto de especificaciones técnicas, administrativas y pedagógicas, que deben evolucionar hacia un estándar de calidad para la educación virtual, que reúna los lineamientos de las organizaciones internacionales y se fortalezca con las necesidades y proyecciones locales. Este es, sin lugar a dudas, un paso significativo en el proceso de buscar mejores niveles de calidad educativa.

\section{Referencias bibliográficas}

Adell, J. Bellver, A \& Bellver C. (2007). Entornos virtuales de aprendizaje y estándares e-Learning. Centre d'Educació i Noves Tecnologies (CENT) Universitat Jaume I.

Agudelo Benjumea, N. M. (2013). Colombia Aprende. Recuperado de: http://aprendeenlinea.udea. edu.co/lms/men/docsoac3/ 0301_metadatos. pdf. 
Aldana, M. F. (2003). Metodología para la construcción de Ambientes Virtuales como soporte para la Educación presencial en la Universidad de los Andes. Ingenieria(18).

Barberá, E. (2008). Aprender eLearning. Fuenlabrada, Madrid, España: Paidós Ibérica.

Carmona Suárez, E. J., \& Gallego Berrío, L. M. (2008). Dashboard digital del docente. Armenia, Quindío: Elizcom.

Carrasco Vargas, S., \& González Escobar, V. (2011). Concepción de e-Learning en el contexto del país: Un uevo concepto de mundo, de empresa y de educación. En C. Rama, \& J. Domínguez Granada, El aseguramiento de la calidad de la educación Virtual. Chimbote, Perú: Gráfica Real.

Facundo, A. (2011). Análisis del aseguramiento de la calidad de la educación superior. En C. Ramos, \& J. Domínguez Granada, El aseguramiento de la calidad de la educación virtual. Chimbote, Perú: Gráfica Real.

Fernández-Majon, M., Moreno, G., Sierra, J., \& Martínez-Ortiz, I. (2007). Uso de estándares aplicados a TIC en Educación. Informe 16 CNICE. España: CNICE. Obtenido de Fernández-Manjón, B., Moreno-Ger, P., Sierra, J. L. y Martínez-Ortíz, I. (2007). Uso de estándares aplicados a TIC en Educación. Informe $\mathrm{N}^{\circ}$ 16. CNICE.

Hilera González, J. R., \& Hoya Marín, R. (2010). Estandares ELearning: Guía de consulta. Alcalá, España: Universidad de Alcalá.

ISO. (2009). International Organization for Standarization. Obtenido de: $\underline{\text { www.iso.org. }}$

Ministerio de Educación Nacional, MEN. (20 de Abril de 2012). Recursos Educativos Abiertos. Bogotá: Colombia: Graficando Servicios Integrados.

Rodríguez, N., \& Sierra, C. (2007). Experiencia de virtualización en la Pontificia Universidad Javeriana de Bogotá. Congreso observatorio para la Cibersociedad, conocimiento abierto y sociedad libre.

Sierra, H. (2009). Modelo pedagógico Humanista tecnológico de la Dirección Nacional de Servicios Académicos Virtuales UN Virtual. Obtenido de www.virtual.unal.edu.co/unv/ portal/articles.

Viscay, C. E. (2005). Los estándares de e-Learning. (U. d. Virtual, Ed.) Ciencia y Tecnología.

Wiley, D. (2007). Estándares de e-learning. Buenas prácticas en e-learing. Universidad a Distancia de Madrid.

Zapata, D. (2003). Contextualización de la enseñanza virtual en la educación superior. Instituto Colombiano para la Educación Superior. 